\title{
Pneumonia and influenza vaccination in rheumatoid arthritis patients
}

\author{
Charles J. Malemud ${ }^{1,2 *}$ \\ ${ }^{1}$ Department of Medicine, Division of Rheumatic Diseases, Case Western Reserve University and University Hospitals Cleveland Medical Center, Cleveland, Ohio \\ USA 44106 \\ ${ }^{2}$ Editor-in-Chief of Global Vaccines and Immunology
}

It is widely accepted that adult patients diagnosed with rheumatoid arthritis (RA) as well as individuals with other autoimmune diseases, such as systemic lupus erythematosus (SLE) and Crohn's disease are at a heightened relative risk for developing pneumococcal pneumonia and viral influenza [1-4]. To examine this problem, Shea et al. analyzed data from 3 large healthcare claims repositories in the United States to analyze rates of pneumonia from all-causes among adults with and without 1 or more of the chronic illnesses currently listed in the Advisory Committee on Immunization Practices' guidelines for indicators of who should receive pneumococcal vaccination and found that rates of all-cause pneumonia among persons with autoimmune diseases, particularly RA, SLE, or Crohn's disease were substantially greater than rates in healthy persons [1]. Based on prior studies Perry et al. also indicated that an optimal time for administering influenza, pneumococcal and human papilloma virus vaccines, as well as others was before initiating non-biologic or biologic disease-modifying antirheumatic drugs (DMARDs) to patients with rheumatic diseases [2]. In a more recent study, Luque Ramos et al. confirmed that the prevalence of pneumonia in Germany requiring hospitalizations were 2-3-fold greater for RA patients compared to an age and sex-matched control group [5]. This association appeared to be especially critical in areas of Germany where vaccination rates tended to be on the low side.

It is also acknowledged that adults who are generally immunocompromised may have a less efficient immune response to vaccination for these infections, yet vaccination rates may be low as a result of anticipating a poor vaccination response $[1,2]$. Indeed, in one study performed by McCarthy et al. in Ireland their results indicated that strategies designed to ensure vaccination compliance was less likely to occur in the setting of a rheumatology clinical practice, not only because of the anticipated poor outcome that was predicted to occur in RA patients, but also as a result of the poor screening that took place prior to vaccination and especially in those patients prior to initiating tumor necrosis factor (TNF)-blockade or other DMARDS [6]. However, they also indicated that influenza vaccines had "good" efficiency when administered to RA patients treated with or without methotrexate (MTX).

Despite some dire predictions regarding vaccine efficacy in RA it appears likely that prophylactic vaccination for preventing pneumococcal pneumonia or influenza in these RA patients should be pro-actively pursued. This assertion should be especially critical because even in the face of widespread use of pneumococcal conjugate vaccines the rate of pneumonia and "invasive" pneumococcal diseases still remains disproportionately high in these "at-risk" patients [1]. However, owing to an anticipated poor outcome that the immune response to vaccine strategies in these RA patients would be feeble when compared to other patient populations has perhaps made vaccination less appealing. But in fact the study results reported by Coulson et al. indicated that a single administration of Pneumovax (PPV23) soon after the diagnosis of RA was confirmed offered up to 10 years of protection against pneumococcal pneumonia even in RA patients who had been treated with MTX and that unvaccinated RA patients in those receiving prednisolone were more likely to have been diagnosed with pneumonia in those previous 10 years [7]. However, this evidence appeared to contradict the results of a prior published study indicating that the treatment of RA patients with MTX and TNF-blockade blunted the responsiveness to vaccination [8]. Additionally this research team also noted that antibody levels in RA patients receiving DMARDs declined at a faster rate implying that re-vaccination should be initiated earlier rather than later in prescribing DMARDs [8]. Furthermore, Brezinschek et al. also reviewed the evidence which showed that the use of "live attenuated vaccines" in RA patients receiving TNF blockade should be strenuously avoided until the overall relative risk for adverse responses to this vaccine strategy was better understood [8].

In order to provide an educated opinion on the extent to which using a vaccination strategy for prevention of pneumonia and/or influenza in RA, it is critical to consider what we know about the immune response to these pneumonia and/or influenza vaccines in RA patients as well as in patients with other autoimmune diseases such as system lupus erythematosus (SLE). In that regard, the results reported by Elkayam et al. may therefore be instructive [9]. This research team reported that all things considered, the results of several clinical trials showed that vaccination with a pneumococcal vaccine did not result in a worsening of clinical symptoms associated with RA or its progression and, furthermore, that administration of this pneumococcal vaccine was associated with an adequate immune response, although one which was lower than that found for vaccination in healthy controls.

So rather than relying on anecdotal evidence from practitioners or individual case reports regarding the clinical efficacy of vaccination

Correspondence to: Charles J. Malemud, $\mathrm{PhD}$, Department of Medicine, Division of Rheumatic Diseases, University Hospital Cleveland Medical Center, Foley Medical Building, Room 207, 2061 Cornell Road, Cleveland, Ohio USA, Tel: (216) 844-7846; E-mail: cjm4@cwru.edu

Key words: rabies vaccines, rabies immunoglobulin, safety, immunogenicity, clinical efficacy

Received: September 20, 2016; Accepted: October 03, 2016; Published: October 06,2016 
in individual RA patients I decided to evaluate the published peerreviewed literature in the PubMed database from 2010 to the present. Here my objective was to consider the details of immune responses in RA patients receiving vaccines employed for preventing pneumonia and influenza since we already know that at least in considering the results from clinical trials the clinical effectiveness of a vaccine may be predicted from understanding the pertinent aspects of the immune response to that vaccine [10]. Therefore I have concluded that through an analysis of these published results one can determine whether or not a solid fundamental foundation exists for establishing the efficacy of vaccination for pneumonia and/or influenza in RA patients, and especially for those RA patients who have been prescribed DMARDs or biologic drugs that are currently employed to treat RA.

To begin, Geometric Mean Titers (GMT) of anti-influenza antibodies were shown to significantly increase for all strains of influenza virus in RA patients treated with DMARDs as well as in healthy control subjects [11]. However, this was not the case in the RA group treated with the B-cell-depleting monoclonal antibody, rituximab, during the early period of therapy for RA. In fact, the GMT of anti-influenza A/ $\mathrm{H} 3 \mathrm{~N} 2$ and $\mathrm{A} / \mathrm{H} 1 \mathrm{~N} 1$ antibodies rose after 28 days of treatment with rituximab although this rise occurred absent elevated numbers of CD19+ B-cells which was compared to CD19+ B-cell levels at the time of vaccination. Most notably, protection and serological conversion was less prominent in the rituximab-treated RA patients than in those RA patients treated with MTX, an indication that rituximab had the capacity to reduce humoral responses following vaccination. However, a moderate restoration of B-cell responses occurred 6-10 months after initiating rituximab-therapy. Importantly, RA did not alter the response to previous vaccination for influenza. Of note, the clinical activity of RA assessed by the Disease Activity Score-28 (DAS28) prior to, and 7 and 28 days after, receiving the influenza vaccination was not influenced by vaccination in either the MTX or rituximab group.

Another study result reported by Arad et al. indicated that CD4+ T-cell immunity to vaccination for influenza was similar in RA patients treated with DMARDs (mostly MTX-treated) and healthy volunteers, whereas humoral immune responses were impaired in these RA patients as assessed by the haemagglutination inhibition assay [12]. Focusing on cellular and/or humoral responses of RA patients to a 7-valent conjugate pneumococcal vaccine in RA patients, Kapetanovic et al. determined that treatment with MTX or TNF blockade or treatment of older patients with this regimen was predictive of an impaired immune response as measured by the "antibody response ratio" [13]. However, dual therapy with either MTX or anti-TNF combined with prednisolone resulted in a more "positive" antibody response. Of note, RA patients treated with the univalent Fc-free PEGylated anti-TNF monoclonal antibody, certolizumab pegol, plus MTX achieved an acceptable humoral response to vaccination with the pneumococcal pneumonia- 23 vaccine and the $\mathrm{H} 1 \mathrm{~N} 1, \mathrm{H} 3 \mathrm{~N} 2 \mathrm{~B} / \mathrm{Brisbane}$ trivalent influenza virus vaccine in an optional 6-month open label extension clinical trial (NCT00993668) suggesting that immune responses to these vaccinations were not impaired [14].

Migita et al. used the multiplex opsonophagocytic killing assay to measure the opsonizing index (a measure of antibody function rather than antibody level) to show that RA patients receiving anti-T-cell proliferation therapy with abatacept plus MTX dual therapy or MTX monotherapy benefited clinically after vaccination with the PPSV23 pneumococcal vaccine [15]. No adverse events were reported with the PPSV23 vaccine although RA patients on the dual therapy produced less IgG antibody than RA patients on methotrexate monotherapy.
However, opsonization responses were preserved. Similar findings were reported in another clinical trial (NCT00828997) where RA patients on various therapies including MTX monotherapy, dual therapy with MTX and TNF-blockade and non-steroidal anti-inflammatory drugs/ analgesics were then vaccinated at the same time therapies were initiated with the 7-valent pneumococcal conjugate vaccine, PCV7. Thus in this clinical trial, vaccinated RA patients developed less serious infections, although antibody titers to serotypes $6 \mathrm{~B}$ and $23 \mathrm{~B}$ were lower in RA patients who developed serious infections [16]. Finally, in a relatively recent study, Bingham III et al. showed that short-term treatment of RA patients with the IL-6 receptor neutralizing monoclonal antibody, tocilizumab, did not cause attenuated humoral responses to PPV23 [17]. However, the 2 basic conclusions drawn from this study were that to maximize responses to Pneumovax (PPV23), RA patients should be current with immunizations prior to initiating tocilizumab therapy and, although most RA patients treated with tocilizumab at a dose of $8 \mathrm{mg} /$ $\mathrm{kg}$ (the highest approved dose) were capable of mounting a detectable immune response to PPV23, the level of protective antibodies were poorly defined [17].

In conclusion, when the results of these published studies and clinical trials are considered taken together, they emphasize that although some RA therapies blunt specific aspects of cellular and humoral responses to pneumococcal and influenza vaccines, the immune response remains strong enough to prevent serious infections from developing in these immunosuppressed individuals.

\section{References}

1. Shea KM, Edelsberg J, Weycker D, Farkouh RA, Strutton DR, et al. (2014) Rates of pneumococcal disease in adults with chronic medical conditions. Open Forum Infect Dis 1: ofu024. [Crossref]

2. Perry LM, Winthrop KL, Curtis JR (2014) Vaccinations for rheumatoid arthritis. Curr Rheumatol Rep 16: 431. [Crossref]

3. Milanovic M, Stojanovich L, Djokovic A, Kontic M, Gvozdenovic E (2013) Influenza vaccination in autoimmune rheumatic disease patients. Tohoku J Exp Med 229: 29-34 [Crossref]

4. Costello R, Winthrop KL, Pye SR, Brown B, Dixon WG (2016) Influenza and pneumococcal vaccination uptake in patients with rheumatoid arthritis treated with immunosuppressive therapy in the UK: A retrospective cohort study using data from the clinical practice research datalink. PLoS One 11: e0153848. [Crossref]

5. Luque Ramos A, Hoffmann F, Callhoff J, Zink A, Albrecht K (2016) Influenza and pneumococcal vaccination in patients with rheumatoid arthritis in comparison with age- and sex-matched controls: results of a claims data analysis. Rheumatol Int 36 : 1255-1263. [Crossref]

6. McCarthy EM, Azeez MA, Fitzpatrick FM, Donnelly S (2012) Knowledge, attitudes and clinical practice of rheumatologists in vaccination of the at-risk rheumatology patient population. J Clin Rheumatol 18: 237-241. [Crossref]

7. Coulson E, Saravanan V, Hamilton J, So KL, Morgan L, et al. (2011) Pneumococca antibody levels after pneumovax in patients with rheumatoid arthritis on methotrexate. Ann Rheum Dis 70: 1289-1291. [Crossref]

8. Brezinschek H-P, Hofstaetter T, Leeb BF, Haindl P, Graninger WB (2008) Immunization of patients with rheumatoid arthritis with antitumor necrosis factor [alpha] therapy and methotrexate. Curr Opin Rheumatol 20: 295-299. [Crossref]

9. Elkayam O, Ablin J, Caspi D (2007) Safety and efficacy of vaccination against streptococcus pneumonia in patients with rheumatic diseases. Autoimmun Rev 6: 312314. [Crossref]

10. Qin L, Gilbert PG, Corey L, McElrath MJ, Self SG (2007) A framework for assessing immunological correlates of protection in vaccine trials. J Infect Dis 196: 1304-1312. [Crossref]

11. Van Assen S, Holvast A, Benne CA, Posthumus MD, van Leeuwen MA, et al. [2010] Humoral responses after influenza vaccination are severely reduced in patients with rheumatoid arthritis treated with rituximab. Arthritis Rheum 62: 75-81. [Crossref]

12. Arad U, Tzadok S, Amir S, Mandelboim M, Mendelson E, et al. [2011] The cellular 
immune response to influenza vaccination is preserved in rheumatoid arthritis patients treated with rituximab. Vaccine 29: 1643-1648. [Crossref]

13. Kapetanovic MC, Roseman C, Jönsson G, Truedsson L, Saxne T, et al. [2011] Antibody response is reduced following vaccination with 7 -valent conjugate pneumococcal vaccine in adult methotrexate-treated patients with established arthritis, but not those treated with tumor necrosis factor inhibitors. Arthritis Rheum 63: 3723-3732. [Crossref]

14. Kivitz AJ, Schechtman J, Texter M, Fichtner A, de Longueville M, et al. [2014] Vaccine responses in patients with rheumatoid arthritis treated with certolizumabpegol: results from a single-blind randomized phase IV trial. J Rheumatol 41: 648-657. [Crossref]

15. Migita K, Akeda Y, Akazawa M, Tohma S, Hirano F, et al. [2015] Effect of abatacept on the immunogenicity of 23-valent pneumococcal polysaccharide vaccination (PPSV23) in rheumatoid arthritis patients. Arthritis Res Ther 17: 357. [Crossref]

16. Nagel J, Geborek P, Saxne T, Jönsson G, Englund M, et al. [2015] The association between antibody levels before and after 7-valent pneumococcal conjugate vaccine immunization and subsequent pneumococcal infection in chronic arthritis patients. Arthritis Res Ther 17: 124. [Crossref]

17. Bingham III CO, Rizzo W, Kivitz A, Hassanali A, Upmanyu R, et al. [2015] Humoral immune response to vaccines in patients with rheumatoid arthritis treated with tocilizumab: results of a randomised controlled trial (VISARA). Ann Rheum Dis 74 818-822. [Crossref]

Copyright: ( $) 2016$ Malemud CJ. This is an open-access article distributed under the terms of the Creative Commons Attribution License, which permits unrestricted use, distribution, and reproduction in any medium, provided the original author and source are credited. 